\title{
Pantoea Infections in the Neonatal Intensive Care Unit
}

\author{
Srinivasan Mani ${ }^{1}$, Jayasree Nair ${ }^{1}$ \\ 1. Pediatrics/Neonatology, State University of New York, Buffalo, USA
}

Corresponding author: Srinivasan Mani, drvazan@gmail.com

\begin{abstract}
Pantoea is a plant pathogen infrequently reported to cause opportunistic bloodstream infections. This gramnegative bacillus is a rare cause of hospital-acquired infections in newborn infants with high mortality. Since the creation of the new genus Pantoea in 1989, the evidence base available to neonatal health care providers is limited. Most of the available literature consists of case reports and case series. This review aims to consolidate the current reported literature on Pantoea infections, focusing on newborn infants and the neonatal intensive care unit (NICU). Prematurity and the associated relative immunocompromised state are major risk factors for hospital-acquired infections due to Pantoea in newborn infants. Recent advances in molecular biology have improved our understanding of the cross-kingdom pathogenesis exhibited by Pantoea. Respiratory symptoms and association with central venous lines are the most common clinical presentation of Pantoea bacteremia in newborn infants. Early institution of appropriate antibiotic therapy against this organism could be lifesaving. Therefore, it is critical for neonatologists to understand the clinical spectrum of Pantoea infections in NICUs.
\end{abstract}

Categories: Pediatrics, Infectious Disease

Keywords: infant, intensive care units, neonatal, opportunistic infections, pantoea

\section{Introduction And Background}

Pantoea, a plant pathogen, is a rare cause of human infections [1]. Pantoea genus was previously included in the Enterobacter genus. This gram-negative bacillus had been reported to cause opportunistic bloodstream infections in neonatal ICUs [2]. Pantoea can cause human infections either as community-acquired infections, including occupational exposures or hospital-acquired infections. Various sites and organs can be affected by Pantoea, causing wound infections, synovitis, septic arthritis, osteomyelitis, bloodstream infections, peritonitis, cholelithiasis, endophthalmitis, endocarditis, dacryocystitis, urinary tract infection, meningitis, brain abscess, and respiratory tract infections. They can also cause allergy and hypersensitivity pneumonitis [3]. In children, especially newborn infants, the infections have a predilection for the respiratory tract, resulting in respiratory failure causing high mortality [4,5].

Review began $11 / 23 / 2020$ Review ended 01/31/2021 Published 02/03/2021

\section{(c) Copyright 2021}

Mani et al. This is an open access article distributed under the terms of the Creative Commons Attribution License CC-BY 4.0., which permits unrestricted use, distribution, and reproduction in any medium, provided the original author and source are credited.

\section{Review \\ Methodology}

We have assimilated research findings from our experience and an extensive review of the literature utilizing key terms in multiple databases, including PubMed, EMBASE, and Science Direct. We used the MeSH terms - "Pantoea," "infant OR newborn," "intensive care, neonatal" for our search. We included "Enterobacter agglomerans" instead of "Pantoea" in our search but did not yield any relevant additional studies to be included. We searched the database up until July 2020.

\section{Epidemiology}

Prevalence

Pantoea infections have been described to cause outbreaks in healthcare settings in the USA since 1970 [6]. The exact prevalence of the disease caused by this pathogen in the NICU is unknown because of the limited literature on this rare pathogen in this patient population. There are few case series reporting systemic infection with this organism in preterm neonates. From the available literature, the prevalence is estimated as low.

In a study of 6,383 patients between the 1st of January 1994 and 1st of June 2005 in the Netherlands, Pantoea colonization was reported in 125 patients (2\%) without clustering [7]. Common sites of colonization include the trachea, urinary tract, and intestinal tract [8]. Another case series noted that five out of 1,665 newborn infants admitted to two different NICUs had nosocomial bloodstream infections due to Pantoea between January 2005 and December 2006 in Kuwait [2]. In a single-center study from Turkey that evaluated the clinical and microbiological characteristics of Pantoea infections in pediatric patients from 2000 - 2015, neonatal infections comprised $34.7 \%$ (eight out of 23 Pantoea isolates) [9]. 


\section{Cureus}

Risk Factors

Prematurity and associated immature immune systems resulting in the relative immunocompromised state are the major risk factors for hospital-acquired infections due to Pantoea in newborn infants [10]. 75\% (30 out of 40) of all neonatal Pantoea infections reported in the English literature as of July 2020 occurred in preterm infants $[1,2,5,7,11-16]$. Most of these infants had at least one comorbid condition. Comorbidities identified in the literature so far include:

1. Prematurity [1]

2. Respiratory distress syndrome $[2,7]$

3. Patent ductus arteriosus [2]

4. Necrotizing enterocolitis [1]

5. Congenital heart disease $[1,7,16]$

6. Intrauterine growth restriction [7]

7. Perinatal Asphyxia [17]

8. Prolonged rupture of membranes [11]

9. Chorioamnionitis [15]

10. VACTERL [17].

Source

Outbreaks of Pantoea associated bloodstream infections in NICUs were related to contamination of parenteral nutrition [17], intravenous fluid [16], infant formula [18], blood products [19], and anesthetic agents [20]. Tracing the origin of infections that occur without clustering in the NICU is challenging. These are defined as 'sporadic' infections. No conclusive evidence for vertical transmission of Pantoea has been documented.

In a single-center retrospective study conducted from 2000-2015 in a tertiary care pediatric hospital in Turkey, the most common specimens from which Pantoea was recovered included pus (six specimens, $42.8 \%$ ), urine (three samples, $21.4 \%$ ), tracheal aspirate (three specimens, $21.4 \%$ ), and blood (three specimens, 21.4\%) from a total of 15 isolates [9]. In newborn infants, blood is the most common sample from which Pantoea is isolated. The usual sources of Pantoea bacteremia include central venous catheters and the respiratory tract. Other causes of Pantoea bacteremia include urinary tract infections, peritonitis, and skin infections (anterior abdominal wall abscess) [1].

\section{Microbiology}

The genus Pantoea was created after Gavini et al. in 1989 proposed the name Pantoea agglomerans to include three similar bacteria, namely Erwinia herbicola, Erwinia milletiae, and Enterobacter agglomerans showing a high level of genomic relatedness found by DNA hybridization [21]. Pantoea is a gram-negative, noncapsulated, non-spore-forming motile bacilli with peritrichous flagella that forms smooth, translucent, and convex colonies in nutrient agar with or without yellow pigmentation. They are facultative anaerobes, oxidase negative. Pantoea can utilize D-xylose, D-ribose, maltose, D-galactose, D-mannose, D-fructose, trehalose, and D-mannitol as carbon sources for energy metabolism [21]. There are 20 species in the genus Pantoea comprised of 13 different DNA hybridization groups [22]. P. agglomerans and P. dispersa were the first two species identified within the genus. $P$. conspicua, $P$. brenneri, $P$. septica, and P. eucrina, P. gaviniae, $P$. calida, $P$. ananatis are the other human pathogenic species isolated from various clinical specimens $[23,24]$. P. agglomerans - $95 \%$ and $P$. dispersa - 5\% constitute the most common species implicated in newborn infants' infections.

Biocontrol Potential of Pantoea

The use of living organisms to control infestations and plant diseases is called biocontrol. Certain Pantoea strains grow as epiphytes and have been shown the potential to decrease the incidence of plant disease. In the United States, the nonpathogenic strains of Pantoea agglomerans are commercially available and used as a biocontrol agent against plant diseases [25]. P. agglomerans E325 has been developed as a commercial biocontrol product for use against a variety of plant diseases [26,27]. 


\section{Pathogenicity}

Attempts made to explain the cross-kingdom pathogenicity of Pantoea spp. were unsuccessful because the plant and clinical isolates were phylogenetically similar and closely related [28,29]. Recent advances in molecular biology have provided whole genome sequences of several Pantoea spp. have helped us reduce the knowledge gap in understanding the determinants of human association of these opportunistic pathogens. The Large Pantoea Plasmid is a candidate plasmid responsible for encoding several proteins that may play a significant role in the colonization, pathogenicity, and antibiotic resistance exhibited by Pantoea spp. in the human host [30]. The hcp and vgrG genomic islands present within the type VI secretory system -1(T6SS-1) locus are the other potential evolutionary hot spots responsible for cross-kingdom pathogenesis [31].

\section{Clinical features}

The most common presentation of Pantoea infections in newborn infants is late-onset sepsis. Outside the NICU outbreak due to contaminated parenteral nutrition reported by Habsah et al. in 2005, there were only two cases of early-onset sepsis so far that has been reported in the neonatal population associated with chorioamnionitis and prolonged rupture of membranes [11,15]. Newborn infants with Pantoea bacteremia present with pulmonary symptoms most commonly. The system-wise clinical features of Pantoea infections reported in the literature so far are included in Table 1.

\begin{tabular}{|l|l|}
\hline System & Clinical presentation \\
\hline Respiratory & $\begin{array}{l}\text { 1. Respiratory distress (most common reported presenting symptom), 2. Pneumonia, 3. Pulmonary hemorrhage, 4. Respiratory } \\
\text { failure }\end{array}$ \\
Cardiac & 1. Septicemic shock - requiring vasoactive agents \\
Neurological & 1. Seizures, 2. Intracranial bleed, 3. Temperature instability/fever (100\% in one case series) \\
Hematological & 1. Leukopenia, 2. Thrombocytopenia, 3. Disseminated intravascular coagulation (DIC) (87.5\% of patients in one case series) \\
Gastrointestinal & 1. Upper gastrointestinal bleeding, 2. Conjugated hyperbilirubinemia \\
Metabolic & 1. Hypoglycemia, 2. Hyperglycemia
\end{tabular}

TABLE 1: Clinical manifestations of Pantoea bacteremia in neonates.

\section{Diagnosis}

Blood culture and isolation of Pantoea is the gold standard in the diagnosis of bloodstream infections. Rapid species-level identification and antibiotic susceptibility are made possible by molecular diagnostic tests like matrix-associated laser desorption/ionization time-of-flight mass spectrometry (MALDI-TOF MS), $16 \mathrm{~s}$ RNA gene sequencing, multi-locus sequence analysis (MLSA), cpn60-based typing. Pantoea infections are relatively rare but potentially dangerous opportunistic pathogens in NICU, especially in preterm infants, so accurate species-level identification and appropriate directed therapy are essential. Rezzonico et al. compared plant-origin and clinical strains of Pantoea spp. in a search for discriminating genotypic/phenotypic markers using multi-locus phylogenetic analysis and fluorescent amplified fragment length polymorphisms fingerprinting. The study found that a large number of clinical isolates from culture collections were found to be improperly designated as P. agglomerans after sequence analysis [32].

A recent study used a combination of MLSA and cpn60-based molecular typing of 54 clinical isolates that had been identified as Pantoea using MALDI-TOF and other clinical typing methods. They showed that $24 \%$ of clinical isolates were misidentified, with MALDI-TOF misidentifying one of every five strains. They found that $P$. agglomerans and $P$. septica are the two correctly identified species [33].

\section{Management}

Antibiotic resistance patterns found in Pantoea limits the choice of antibiotics. According to our best knowledge, there are no clinical studies that have compared the antibiotic regimens against Pantoea. The evidence for the efficacy of the various antibiotic choices in neonates is derived from individual case reports and a few case series.

\section{Antibiotic Resistance}

Pantoea agglomerans have shown resistance to a wide variety of antibiotics, including early-generation penicillin, early-generation cephalosporins, broad-spectrum cephalosporins, and antipseudomonal penicillins, fluoroquinolones, aminoglycoside, TMP-SMX, and tetracyclines. 
In a study evaluating powdered infant formula milk samples between 2011 and 2012, P. agglomerans were isolated from $6.4 \%$ of samples. The isolates showed susceptibility to tigecycline, chloramphenicol, cefepime, levofloxacin, minocycline, and colistin. Fifty percent of the strains in that study were resistant to cefotaxime, moxifloxacin, cotrimoxazole, and ticarcillin [18].

In a case series that included pediatric and newborn infants, all isolates of $P$. agglomerans showed antimicrobial susceptibility to amikacin, gentamicin, meropenem, and trimethoprim-sulfamethoxazole and $92.5 \%$ of isolates were susceptible to broad-spectrum cephalosporins and semisynthetic penicillin, $62.3 \%$ to extended-spectrum cephalosporins, and only $47.2 \%$ to ampicillin [1].

In another study that evaluated the antimicrobial susceptibility of $P$. agglomerans over 15 years in pediatric patients, $21.4 \%$ of isolates showed carbapenem resistance [9]. The use of broad-spectrum antibiotics is known to increase the risk of colonization in hospital settings and increase the risk of the emergence of antibiotic resistance. The gastrointestinal colonization and later translocation may act as a reservoir for the organism [34].

Mechanism of Drug Resistance

The drug resistance genes identified in Pantoea spp. include CTX-M-15, TEM-1, dfrA14, tet(K), qnrB1, aac69-16-cr, class1 and class 2 integron, aph, aadA1, cat1, qacDE, Sul1 [35].

CTX-M-15 is the most common, widely prevalent ESBL gene studied concerning the extraintestinal pathogenic E. coli ST131 [36]. Amp C beta-lactamase, another important ESBL gene common with Enterobacter aerogenes, another important saprophytic gram-negative bacilli, has not been isolated from Pantoea spp. Plasmid and integron harbor the candidate genes involved in drug resistance associated with Pantoea spp.

Treatment

Success in managing Pantoea bloodstream infections in newborn infants depends on prompt identification and early administration of appropriate antibiotic therapy combined with routine supportive care. Since most of the reported Pantoea spp. BSI in newborns has been associated with central venous lines; care should be taken to identify this association.

\section{Antimicrobial selection}

First-Line Therapy

We suggest that the neonatologist pay attention to the site of infection, gestational age of the infant, and their comorbidities while choosing the antimicrobial treatment. For term or late preterm infants with infections occurring without central venous lines or other comorbidities, the aminoglycoside (gentamicin or amikacin) combined with ampicillin is the appropriate first-line therapy [15].

In a case series that had reported positive outcomes in very preterm infants with Pantoea BSI in the presence of central venous lines and comorbidities with or without hemodynamic comprise, broad-spectrum antibiotics like a combination of third-generation cephalosporin with aminoglycosides or carbapenems or ureidopenicillin/ beta-lactamase were used as the first line of therapy [2].

Based on our experience (unpublished data) with this organism as an etiological agent causing late-onset sepsis in extremely preterm infants with comorbidities (ventilator-dependent bronchopulmonary dysplasia and central venous catheter), we recommend carbapenems as first-line therapy for pneumonia or bacteremia. For uncomplicated infections limited to the genitourinary tract, aminoglycosides could be used as the first-line therapy.

Alternative Therapy

For Pantoea strains resistant to carbapenems, trimethoprim/ sulfamethoxazole can be a good alternative. This medication in neonates requires regular monitoring of serum bilirubin levels and liver enzymes during the entire course of therapy.

\section{Prognosis}

Pantoea bloodstream infection being an opportunistic pathogen affecting infants with comorbidities, has a high mortality rate. The mortality rate is exceptionally high in preterm infants. The reported mortality rate among the case series and case reports of Pantoea infections in the NICU is $45 \%$ (18/40). More than $95 \%$ of those infants were premature at birth. The most common cause of mortality is respiratory failure and septic shock. In one case series, including five preterm infants with Pantoea BSI who were started on meropenem or piperacillin/tazobactam as first-line antibiotics had a favorable outcome of survival in all of them [2]. On 
the contrary, the exceptionally high mortality rates of $87.5 \%(7 / 8)[17]$ and $100 \%(3 / 3)[7]$ have been reported in case series describing Pantoea outbreaks in NICUs.

\section{Conclusions}

Pantoea is a relatively rarer but potentially dangerous hospital-acquired infection encountered in NICUs, especially in preterm infants with comorbid conditions. P. agglomerans and P. dispersa are the most common pathogenic species in newborns. Colonization tends to happen in the respiratory tract, genitourinary tract, and gastrointestinal tract. Translocation into the bloodstream produces aggressive infection. Sensitivity to routine first-line antibiotics is variable with a high prevalence of multidrug resistance. In this scenario, neonatologists should clearly understand this pathogen, which would help treat the neonates with this infection efficiently. With limited available literature, multicenter data collection may facilitate more extensive studies that could yield valuable information to assist in the clinical management of Pantoea infections.

\section{Additional Information \\ Disclosures}

Conflicts of interest: In compliance with the ICMJE uniform disclosure form, all authors declare the following: Payment/services info: All authors have declared that no financial support was received from any organization for the submitted work. Financial relationships: All authors have declared that they have no financial relationships at present or within the previous three years with any organizations that might have an interest in the submitted work. Other relationships: All authors have declared that there are no other relationships or activities that could appear to have influenced the submitted work.

\section{References}

1. Cruz AT, Cazacu AC, Allen CH: Pantoea agglomerans, a plant pathogen causing human disease . J Clin Microbiol. 2007, 45:1989-1992. 10.1128/JCM.00632-07

2. Aly NY, Salmeen HN, Lila RA, Nagaraja PA: Pantoea agglomerans bloodstream infection in preterm neonates. Med Princ Pract. 2008, 17:500-503. 10.1159/000151575

3. Mackiewicz B, Dutkiewicz J, Siwiec J, et al.: Acute hypersensitivity pneumonitis in woodworkers caused by inhalation of birch dust contaminated with Pantoea agglomerans and Microbacterium barkeri. Ann Agric Environ Med. 2019, 26:644-655. 10.26444/aaem/114931

4. Lee NE, Chung IY, Park JM: A case of Pantoea endophthalmitis. Korean J Ophthalmol. 2010, 24:318-321. 10.3341/kjo.2010.24.5.318

5. Van Rostenberghe H, Noraida R, Wan Pauzi WI, et al.: The clinical picture of neonatal infection with Pantoea species. Jpn J Infect Dis. 2006, 59:120-121.

6. Mackel DC, Maki DG, Anderson RL, Rhame FS, Bennett JV: Nationwide epidemic of septicemia caused by contaminated intravenous products: mechanisms of intrinsic contamination. J Clin Microbiol. 1975, 2:486497.

7. Bergman KA, Arends JP, Scholvinck EH: Pantoea agglomerans septicemia in three newborn infants . Pediatr Infect Dis J. 2007, 26:453-454. 10.1097/01.inf.0000261200.83869.92

8. Rizi KS, Farsiani H, Hiradfar S, et al.: Pantoea agglomerans septicemia in infants: the first case report from Iran and review of literature. Acta Scientific Microbiology. 2019/8/19. 2019, 2:114-117. 10.31080/ASMI.2019.02.0346

9. Büyükcam A, Tuncer Ö, Gür D, et al.: Clinical and microbiological characteristics of Pantoea agglomerans infection in children. J Infect Public Health. 2018, 11:304-309. 10.1016/.j.jiph.2017.07.020

10. Melville JM, Moss TJ: The immune consequences of preterm birth . Front Neurosci. 2013, 7:79. 10.3389/fnins.2013.00079

11. Lalas KM, Erichsen D: Sporadic Pantoea agglomerans bacteremia in a near-term female: case report and review of literature. Jpn J Infect Dis. 2010, 63:290-291.

12. Mehar V, Yadav D, Sanghvi J, Gupta N, Singh K: Pantoea dispersa: an unusual cause of neonatal sepsis. Braz J Infect Dis. 2013, 17:726-728. 10.1016/j.bjid.2013.05.013

13. Segado-Arenas A, Alonso-Ojembarrena A, Lubian-Lopez SP, Garcia-Tapia AM: Pantoea agglomerans: a new pathogen at the neonatal intensive care unit?. Arch Argent Pediatr. 2012, 110:e77-e79. 10.5546/aap.2012.e77

14. Senanayake N, Thevanesam V, Karunanayake L: An outbreak of Pantoea agglomerans infection in the neonatal intensive care unit at Teaching Hospital, Kandy, Sri Lanka. Sri Lanka J Child Health. 2016, 45:3233. 10.4038/sljch.v45i1.8082

15. Tiwari S, Beriha SS: Pantoea species causing early onset neonatal sepsis: a case report . J Med Case Rep. 2015, 9:188. 10.1186/s13256-015-0670-0

16. Matsaniotis NS, Syriopoulou VP, Theodoridou MC, Tzanetou KG, Mostrou GI: Enterobacter sepsis in infants and children due to contaminated intravenous fluids. Infect Control. 1984, 5:471-477. 10.1017/S0195941700060872

17. Habsah H, Zeehaida M, Van Rostenberghe H, et al.: An outbreak of Pantoea spp. in a neonatal intensive care unit secondary to contaminated parenteral nutrition. J Hosp Infect. 2005, 61:213-218. 10.1016/j.jhin.2005.01.004

18. Mardaneh J, Dallal MMS: Isolation, identification and antimicrobial susceptibility of Pantoea (Enterobacter) agglomerans isolated from consumed powdered infant formula milk (PIF) in NICU ward: First report from Iran. Iran J Microbiol. 2013, 5:263-267.

19. Boszczowski I, Nóbrega de Almeida Júnior J, Peixoto de Miranda EJ, et al.: Nosocomial outbreak of Pantoea 
agglomerans bacteraemia associated with contaminated anticoagulant citrate dextrose solution: new name, old bug?. J Hosp Infect. 2012, 80:255-258. 10.1016/j.jhin.2011.12.006

20. Bennett SN, McNeil MM, Bland LA, et al.: Postoperative infections traced to contamination of an intravenous anesthetic, propofol. N Engl J Med. 1995, 333:147-154. 10.1056/NEJM199507203330303

21. Gavini F, Mergaert J, Beji A, et al.: Transfer of Enterobacter agglomerans (Beijerinck 1888) Ewing and Fife 1972 to Pantoea gen. nov. as Pantoea agglomerans comb. nov. and Description of Pantoea dispersa sp. nov. Int J Syst Evol Microbiol. 1989, 39:337-345. 10.1099/00207713-39-3-337

22. Walterson AM, Stavrinides J: Pantoea: insights into a highly versatile and diverse genus within the Enterobacteriaceae. FEMS Microbiol Rev. 2015, 39:968-984. 10.1093/femsre/fuv027

23. Brady CL, Cleenwerck I, Venter SN, Engelbeen K, De Vos P, Coutinho TA: Emended description of the genus Pantoea, description of four species from human clinical samples, Pantoea septica sp. nov., Pantoea eucrina sp. nov., Pantoea brenneri sp. nov. and Pantoea conspicua sp. nov., and transfer of Pectobacterium cypripedii (Hori 1911) Brenner et al. 1973 emend. Hauben et al. 1998 to the genus as Pantoea cypripedii comb. nov. Int J Syst Evol Microbiol. 2010, 60:2430-2440. 10.1099/ijs.0.017301-0

24. Popp A, Cleenwerck I, Iversen C, De Vos P, Stephan R: Pantoea gaviniae sp. nov. and Pantoea calida sp. nov., isolated from infant formula and an infant formula production environment. Int J Syst Evol Microbiol. 2010, 60:2786-2792. 10.1099/ijs.0.019430-0

25. Morin A. Pantoea. In: , editors: Encyclopedia of Food Microbiology (Second Edition), pp 1028-1032 . Batt CA, Tortorello ML (ed): Academic Press, Oxford; 2014.

26. Pusey PL: Biological control agents for fire blight of apple compared under conditions limiting natural dispersal. Plant Dis. 2002, 86:639-644. 10.1094/PDIS.2002.86.6.639

27. Pusey PL, Stockwell VO, Reardon CL, Smits TH, Duffy B: Antibiosis activity of Pantoea agglomerans biocontrol strain E325 against Erwinia amylovora on apple flower stigmas. Phytopathology. 2011, 101:12341241. 10.1094/PHYTO-09-10-0253

28. Völksch B, Thon S, Jacobsen ID, Gube M: Polyphasic study of plant- and clinic-associated Pantoea agglomerans strains reveals indistinguishable virulence potential. Infect Genet Evol. 2009, 9:1381-1391. 10.1016/j.meegid.2009.09.016

29. Nadarasah G, Stavrinides J: Quantitative evaluation of the host-colonizing capabilities of the enteric bacterium Pantoea using plant and insect hosts. Microbiology. 2014, 160:602-615. 10.1099/mic.0.073452-0

30. De Maayer P, Chan WY, Blom J, et al.: The large universal Pantoea plasmid LPP-1 plays a major role in biological and ecological diversification. BMC Genomics. 2012, 13:625. 10.1186/1471-2164-13-625

31. De Maayer P, Venter SN, Kamber T, Duffy B, Coutinho TA, Smits THM: Comparative genomics of the type VI secretion systems of Pantoea and Erwinia species reveals the presence of putative effector islands that may be translocated by the VgrG and Hcp proteins. BMC Genomics. 2011, 12:576. 10.1186/1471-2164-12576

32. Rezzonico F, Smits TH, Montesinos E, Frey JE, Duffy B: Genotypic comparison of Pantoea agglomerans plant and clinical strains. BMC Microbiol. 2009, 9:204. 10.1186/1471-2180-9-204

33. Soutar CD, Stavrinides J: Molecular validation of clinical Pantoea isolates identified by MALDI-TOF . PLoS One. 2019, 14:e0224731. 10.1371/journal.pone.0224731

34. Cheng A, Liu CY, Tsai HY, et al.: Bacteremia caused by Pantoea agglomerans at a medical center in Taiwan, 2000-2010. J Microbiol Immunol Infect. 2013, 46:187-194. 10.1016/j.jmii.2012.05.005

35. Raphael E, Riley LW: Infections caused by antimicrobial drug-resistant saprophytic gram-negative bacteria in the environment. Front Med. 2017, 4:183. 10.3389/fmed.2017.00183

36. Nicolas-Chanoine M-H, Blanco J, Leflon-Guibout V, et al.: Intercontinental emergence of Escherichia coli clone O25:H4-ST131 producing CTX-M-15. J Antimicrobial Chemother. 2007, 61:273-281.

$10.1093 / \mathrm{jac} / \mathrm{dkm} 464$ 